\title{
Influencia de los observadores sobre la agresión y el sentimiento de maltrato escolar
}

\author{
Sara CONDE VÉLEZ y Jose Antonio ÁVILA FERNANDEZ
}

Universidad de Huelva, Departamento de Educación, España

(Recibido, 13 de Octubre, 2017; Aceptado, 22 de Mayo, 2018)

RESUMEN: El objetivo del trabajo que se presenta es analizar la influencia que pueden ejercer los observadores sobre el sentimiento de agresor o víctima a partir de los datos ofrecidos por el estudio realizado sobre el acoso escolar, desde un análisis estructural, en los centros de Educación Primaria públicos de la provincia de Huelva (España). Se elaboran tres escalas ad hoc: «Víctimas», «Agresor» y «Observador» aplicándose a una muestra de 2.156 sujetos pertenecientes a treinta centros de Educación Infantil y Primaria de titularidad pública de la provincia de Huelva. Respecto al género, el 51,4\% del alumnado son niños y el 48,6\% son niñas. Las edades están comprendidas entre 8 y 13 años. El alumnado se distribuye respetando la formación natural de los grupos por clases, esto es, alumnado que cursan tercero (532), cuarto (574), quinto (532) y sexto (518) curso de Educación Primaria. No se encuentran diferencias estadísticamente significativas con respecto al sexo $\left(X^{2}=1.67, p=.196\right)$, ni edad $(\mathrm{t}=.589, \mathrm{p}=.55)$. Para el tratamiento de los datos se utiliza la técnica Partial Least Squares (PLS). Los resultados confirma la influencia que tiene la presencia de los observadores sobre la agresión, en sus distintas manifestaciones y sobre el sentimiento de maltrato. El modelo propuesto pone de relieve que el acoso escolar no sólo consiste en peleas y golpes, sino que el hostigamiento y el maltrato verbal, se presenta como el tipo de violencia más habitual.

Palabras clave: Agresión, maltrato escolar, observadores, prevención.

\section{Influence of bystanders on aggression and a sense of school bullying}

ABSTRACT: The concern of the work presented is to analyze the influence they can exert observers on the feeling of aggressor or victim from data provided by the study of bullying, from a structural analysis public centers Education primary province of Huelva (Spain). "Victims", "Aggressor" and "Observer" applied to a sample of 2,156 individuals belonging to thirty centers and Primary Education: three scales are developed ad hoc. Regarding gender, $51.4 \%$ of students are children and $48.6 \%$ are girls. The ages are between 8 and 13 years. The students are distributed respecting the natural formation of the groups by class, that is, students who attend third (532), fourth (574), fifth (532) and sixth (518) Primary Education course. No statistically significant differences were found with respect to sex $\left(\mathrm{X}^{2}=.67, \mathrm{p}=.196\right)$, nor age $(\mathrm{t}=.589, \mathrm{p}=.55)$. For data processing technique Partial Least Squares (PLS) is used. The results confirmed the influence of the presence of observers on aggression, in its various manifestations and the feeling of abuse. The proposed model emphasizes that bullying is not 
just about fights and beatings, but harassment and verbal abuse, is presented as the most common type of violence.

Keywords: Aggression, school bullying, bystanders, prevention.

Correspondencia: Sara Conde Vélez. Universidad de Huelva, Departamento de Educación, Campus "El Carmen”, 21771 - Huelva. E-mail: sara.conde@,dedu.uhu.es

\section{Introducción}

El acoso escolar, cuya existencia ha sido comprobada en múltiples contextos y países, se ha convertido en un problema frecuente en las escuelas, independientemente de la calidad de las instituciones públicas o privadas (Cuevas \& Marmolejo, 2016).

Los alumnos implicados en problemas de relación en el ámbito escolar se pueden agrupar, fundamentalmente, en víctimas, agresores y observadores (Garaigordobil, 2017, González, Gutiérrez \& Checa, 2017). Cada uno de estos grupos se subdivide según la implicación en los hechos o sus formas de intervención. El rol del alumno/a agresor/a se describe como un escolar, chico o chica, que posee poca conciencia moral sobre sus actos, tiene capacidad para autoexculparse y cierta popularidad entre sus compañeros. En general, diversos autores (Garaigordobil, Martínez-Valderrey, \& Machimbarrena, 2017; Ovejero, Smith \& Yubero, 2013; Paulino, Avilés \& Sales, 2016) sostienen que el agresor:

- Suele ser un sujeto dominante, exaltado e impulsivo.

- Carece de empatía para aquellos con los que se relaciona.

- No le gusta seguir normas establecidas y se rebela contra ellas.

- Su comportamiento violento es habitual y supone un beneficio para el fin de su comportamiento.

- Físicamente es más fuerte que sus víctimas.

- Son identificados como orgullosos e indiferentes.

Por otra parte, la víctima se describe como chico o chica, más débil, con pocas habilidades sociales, bajos niveles de popularidad, no teniendo relevancia su situación en el rendimiento académico e incapaz de afrontar el ataque al que es sometido (Cerezo et al., 2015). Se establecen dos tipos de víctima: por una parte, la activa, provocadora al exhibir su debilidad, facilitando las acciones de agresión y, por otra parte, la pasiva, que no exterioriza su personalidad y sufre, como consecuencia, en silencio los efectos de la intimidación (Puértolas \& Montiel, 2017).

Melero (2017) señala en una reciente investigación las importantes consecuencias que genera el acoso escolar en las víctimas tanto a nivel académico, psicológico y social.

Respecto a los observadores, cabe resaltar, el papel que juegan en el inicio y mantenimiento del acoso escolar, no solamente constituyen la proporción mayoritaria de participantes en el fenómeno, sino que también incluyen un amplio rango de posibles roles que, dependiendo de las acciones a favor o en contra de víctimas e intimidadores, refuerzan, incentivan, rechazan o ignoran el acoso escolar (Cuevas \& Marmolejo, 2016).

Peets et al. (2015), destacan la importancia de las emociones, en la defensa del comportamiento, que provoca el observar la difícil situación de la víctima en una situación de acoso escolar, nivel de empatía (Song \& Oh, 2017). Sin embargo, es poco probable que la empatía afectiva sea suficiente, como único elemento motivador, para defender el comportamiento. También es importante que los niños se sientan seguros de su capacidad para defender a la víctima.

(c) Psy, Soc, \& Educ, 2018, Vol. 10(2) 
El enfoque del rol participante en el observador ha arrojado luz sobre las diferentes formas en que los miembros de un grupo, como un aula, contribuyen al acoso. Más allá de la díada intimidación-víctima, los incidentes de intimidación, generalmente, involucran a un grupo de espectadores: los asistentes se unen y ayudan al cabecilla a intimidar, mientras que los reforzadores permanecen alrededor y señalan su aprobación del acoso con risas o vítores.

Los defensores intervienen para interponerse en nombre de los compañeros victimizados o apoyar a las víctimas, mientras que los forasteros permanecen pasivos y no dan señal explícitamente de aprobación o desaprobación de la situación (Saarento \& Salmivalli, 2015).

El alumnado expuesto a acciones de agresividad entre iguales adquiere un aprendizaje que le puede llevar a valorar positivamente al agresor por su posición superior ante los demás e, incluso, asumir el comportamiento como forma de afrontar dificultades relacionales.

Salmivalli (2013:122) afirma que "los niños y adolescentes que afrontan problemas de acoso como espectadores están atrapados en un dilema social. Por un lado, entienden que el acoso está mal y les gustaría hacer algo para pararlo, pero, por otro, se esfuerzan para asegurarse su propio estatus y su seguridad en el grupo de iguales".

Por otro lado, identificar tipos de acoso ha sido otro de los aspectos en lo que se han centrado las investigaciones sobre la temática. Menesini \& Salmivalli (2017) establecen que la intimidación comprende desde ataques verbales (insultos, amenazas), comportamientos físicos (golpear, patear, dañar la propiedad de la víctima) y agresión relacional/social (exclusión, difusión de rumores) hasta las formas más recientes de ataques a través de Internet y las nuevas tecnologías (cyberbullying).

Investigaciones recientes han demostrado una cierta superposición entre el acoso escolar y el cyberbullying, ya que los menores que están siendo acosados en la vida real tienden a ser intimidados al mismo tiempo a través de los dispositivos digitales (Ortega-Ruiz, Del Rey \& Casas, 2016).

Según la Organización Mundial de la Salud (2016), en adelante OMS, "la violencia es el uso intencional de fuerza o poder, amenazado o real, contra uno mismo, contra otra persona o contra un grupo o comunidad, que da como resultado lesiones, muerte, daño psicológico, mal desarrollo o privación". Por lo tanto, esta definición de violencia abarca muchos más hechos que el daño físico. Sus consecuencias son mucho más amplias que las muertes y lesiones, y puede incluir enfermedades transmisibles y no transmisibles, daños psicológicos, conductas de riesgo, bajo rendimiento educativo y ocupacional y participación en el crimen.

Esta clasificación de actos violentos, en la que se pretende concentrar todos los posibles, teniendo en cuenta la naturaleza de la violencia y el autor del acto violento. A su vez, se subdividen en tipos de violencia más específicos: Ideación suicida y Cutting, en la dirigida contra uno mismo; el maltrato (incluido el castigo violento) involucra violencia física, sexual y psicológica / emocional, el Bullying (incluido el acoso cibernético, Zych, Ortega-Ruiz \& Marín-López, 2016), violencia juvenil (incluye asalto físico con armas o sin armas, y puede involucrar a pandillas), Violencia en la pareja (o violencia doméstica), Violencia Sexual y Violencia emocional o psicológica (incluye restringir los movimientos de un niño, denigración, ridículo, amenazas e intimidación, discriminación, rechazo y otras formas no físicas de tratamiento hostil. La violencia puede involucrar obligar a un niño a observar un acto de violencia, o ser testigo incidental de violencia entre dos o más personas. 
Hay muchos estudios realizados sobre el acoso escolar pero ahora los investigadores sostienen que se hace necesario examinar el rol de los observadores, dada la escasez de investigaciones realizadas que ilustren de manera específica su relevancia dentro del fenómeno (Cuevas \& Marmolejo, 2016; Garaigordobil, 2017).

La preocupación del trabajo que se presenta es la de analizar la influencia que pueden ejercer los observadores sobre el sentimiento de agresor o víctima a partir de los datos ofrecidos por el estudio realizado sobre el acoso escolar, desde un análisis estructural, en los centros públicos de Educación Primaria de la provincia de Huelva.

\section{Método}

El objetivo de esta investigación consiste en analizar la influencia que ejercen los observadores sobre el sentimiento de agresor o víctima.

Para ello el modelo de investigación que se diseña plantea el análisis de la relación de los indicadores asociados al sentimiento de maltrato escolar (víctima) con otras variables de conductas agresivas. Por otro lado, se propone en el modelo, el estudio de la influencia que ejercen los observadores sobre las conductas agresivas (agresores, autores del acoso a un igual) y sobre el sentimiento de maltrato (víctimas, receptores de la agresión) (ver Figura 1).

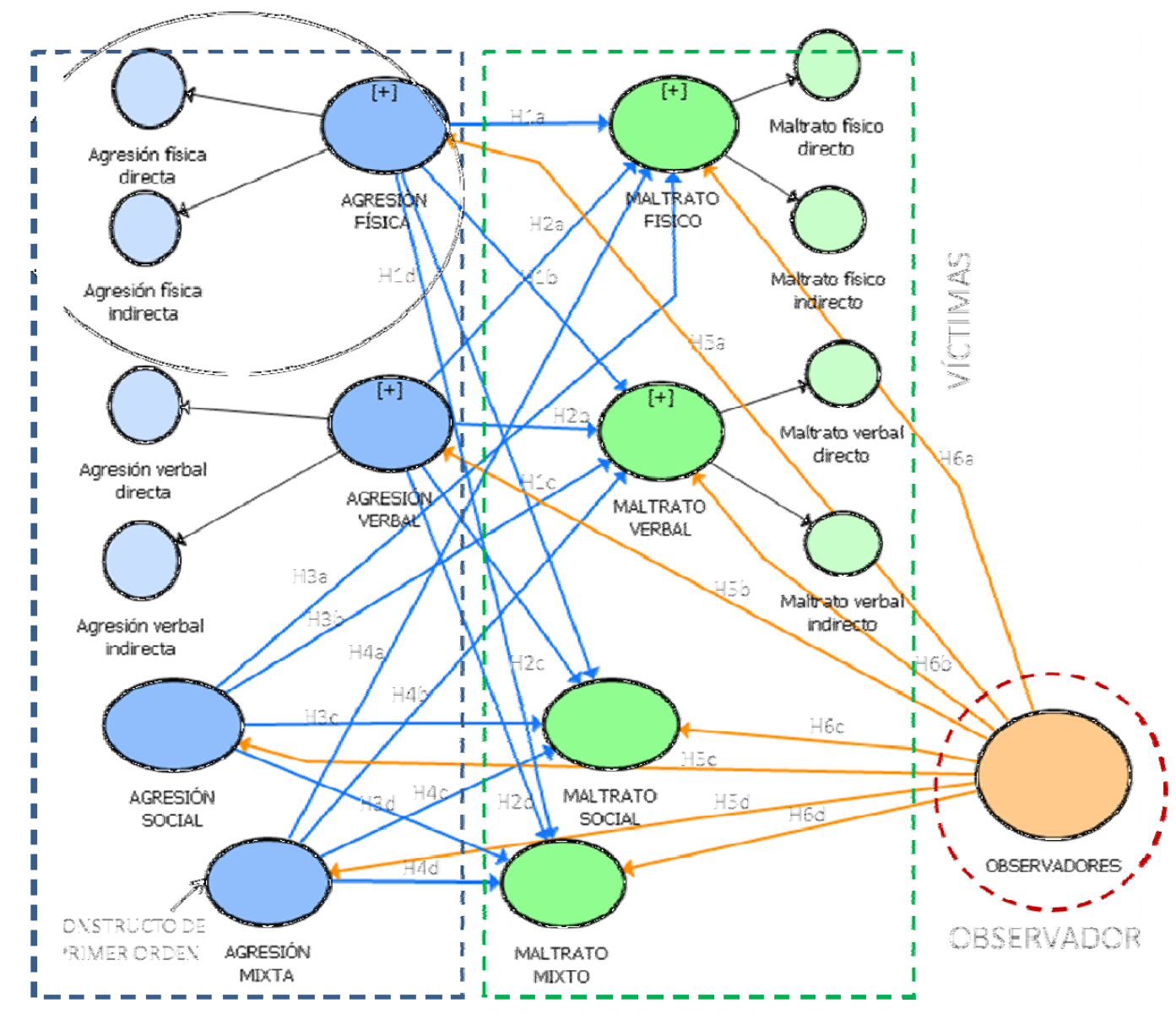

Figura 1. Modelo de las relaciones propuesto.

Fuente: Gráfico obtenido con el programa SmartPLS 2.0. 
El modelo está conformado por un total de nueve constructos, cinco de primer orden (variables latentes) y cuatro de segundo orden; los constructos de segundo orden poseen diferentes dimensiones (variables latentes) de primer orden para identificar correctamente dicho modelo, es decir trata de describir diferentes factores de un constructo; cada dimensión posee sus propias medidas o indicadores. En el modelo se observa una variable predictora (independiente) «observadores» y ocho variables criterio (dependiente).

\section{Muestra}

La muestra final asciende a 2.156 sujetos, pertenecientes a treinta centros de Educación Infantil y Primaria públicos de la provincia de Huelva, correspondientes a las seis comarcas en que ésta se divide y se distribuyen en veinticuatro localidades.

La muestra de alumnado participante en la investigación se corresponde con aquéllos que cursan tercero (532), cuarto (574), quinto (532) y sexto (518) curso de Educación Primaria. El alumnado se distribuye respetando la formación natural de los grupos por clases. Con respecto al género, el 51,4\% del alumnado son niños y el 48,6\% son niñas. Las edades están comprendidas entre 8 y 13 años $(\mathrm{M}=10.05$; $\mathrm{DT}=1.31$; Moda=10; Mediana=10). No se encuentran diferencias estadísticamente significativas con respecto al sexo $\left(X^{2}=1.67, p=.196\right)$, ni edad $(\mathrm{t}=.589, \mathrm{p}=.55)$.

Para la selección de la muestra se ha utilizado un muestreo incidental en los que se utilizan los elementos de la población más fácilmente accesibles.

\section{Instrumento}

Para la recogida de datos se elaboran tres escalas ad hoc: «Víctimas», «Agresor»y «Observador». Cada una de las mismas queda integrada por una serie de indicadores a valorar a través de una escala tipo Likert con valores comprendidos entre 1 y 4, siendo 1 nunca, 2 alguna vez, 3 muchas veces y 4 siempre. Las escalan han sido elaboradas a partir del estudio de un importante número de cuestionarios de autores diversos (Albaladejo et al., 2013; Gómez Bahillo, 2006; Collell \& Escudé, 2003; Magaz et al., 2016; Lera \& Olías, 2002; Ortega, Del Rey \& Mora-Merchán, 2008). En un primer momento, se pasó a una muestra piloto compuesta por alumnado de las edades a las que se dirigía la investigación (24 sujetos), obteniendo un Alfa de Cronbach de 0,838.

En la Tabla 1 se muestran las escalas utilizadas en relación con los distintos sujetos implicados en el acoso escolar. Se solicitan datos desde la posición de víctima, agresor y observador. 
Tabla 1. Escalas sujetos implicados en el acoso escolar: victimas, agresores y observadores

\begin{tabular}{l}
\hline VÍCTIMAS \\
\hline 15. Amenazar \\
16. Pegar (golpes, patadas, puñetazos, zancadillas) \\
17. Esconder cosas \\
18. Romper materiales (la mochila, los cuaderno) \\
19. Quitar cosas \\
20. Insultar, hacer burlas \\
21. Ofender (decir motes) \\
22. Hablar mal (calumniar) \\
23. Dejar solo/a, para hacer daño \\
24. No dejar participar en el juego, no querer jugar conmigo \\
25. No querer estudiar conmigo (rechazarme) \\
26. Obligar a hacer algo que yo no quiero
\end{tabular}

AGRESOR/A

28. Amenazar

29. Pegar (golpes, patadas, puñetazos, zancadillas)

30. Esconder cosas

31. Romper materiales (mochilas, cuadernos, libros...)

32. Quitar cosas

33. Insultar, hacer burlas

34. Ofender (decir motes)

35. Hablar mal (calumniar)

36. Dejar solo/a, para hacer daño

37. No dejar participar en el juego, no dejar jugar conmigo

38. No dejar estudiar conmigo (rechazar)

39. Obligar a hacer algo que quiero conseguir

\section{OBSERVADOR/A}

41. Amenazar

42. Pegar (golpes, patadas, puñetazos, zancadillas)

43. Esconder cosas

44. Romper materiales (mochilas, cuadernos, libros...)

45. Quitar cosas

46. Insultar, hacer burlas

47. Ofender (decir motes)

48. Hablar mal (calumniar)

49. Dejar solo/a, para hacer daño

50. No dejar participar en el juego, no dejar jugar

51. No dejar estudiar (rechazar)

52. Obligar a hacer algo que no quieren

Fuente: Elaboración propia. 


\section{Análisis de datos}

Para el análisis de datos, se ha recurrido a la técnica Partial Least Squares (PLS en adelante) permitiendo incorporar constructos abstractos e inobservables medidos por medio de indicadores.

\section{Resultados}

\section{Análisis e interpretación del modelo}

En una primera fase se propone un ajuste del modelo interpretando la regresión de todos los constructos de conductas agresivas relacionándolos con las distintas manifestaciones de maltrato, los resultados obtenidos se ajustaron discretamente a los datos del modelo. Tras eliminar aquellas estimaciones no significativas se hallan mejores valores de ajuste que no contradicen las premisas teóricas del modelo (Figura 2).

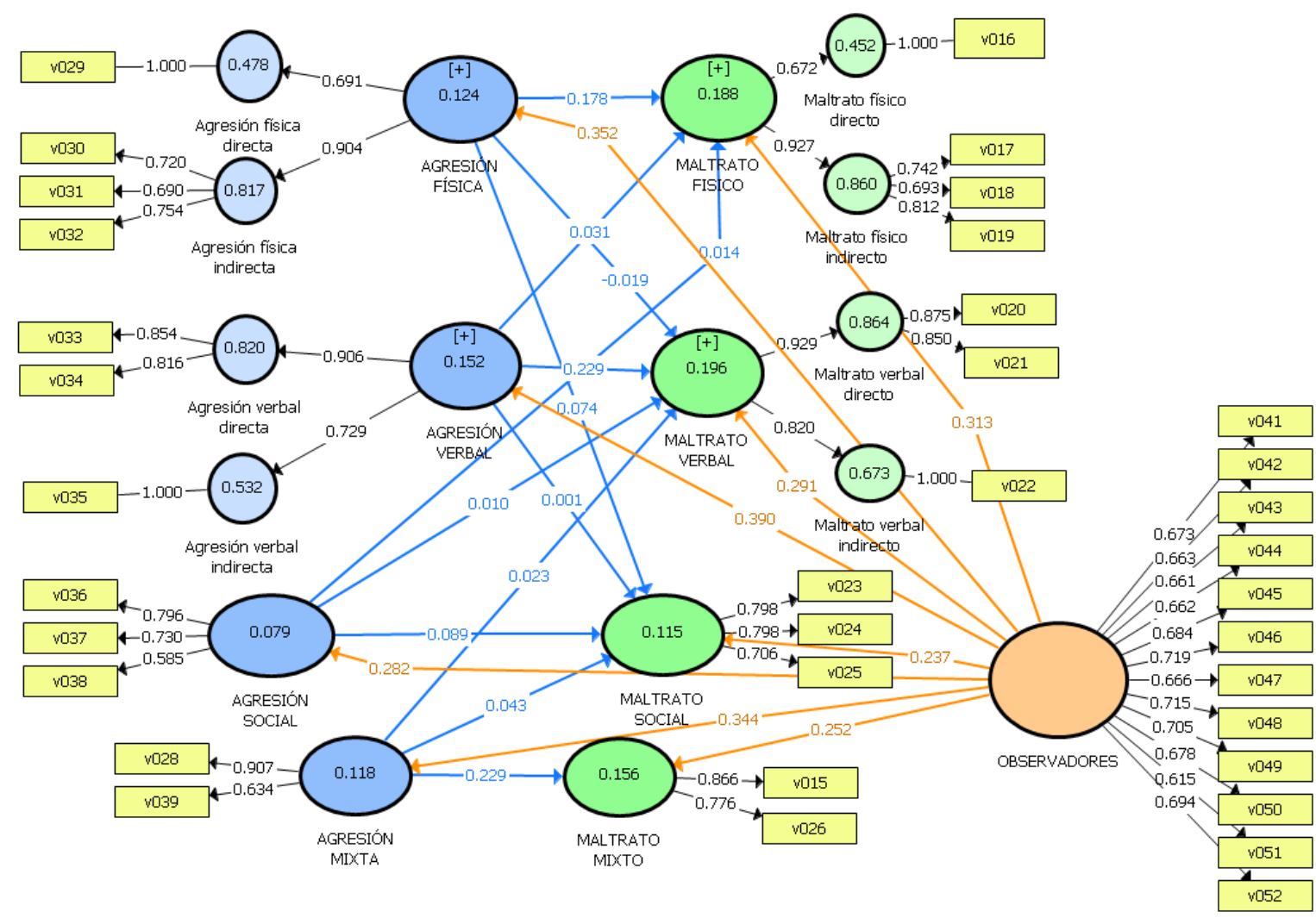

Figura 2. Estimaciones del modelo de relaciones propuesto.

Fuente: Gráfico obtenido con el programa SmartPLS 2.0.

\section{Valoración del modelo de medida}

La fiabilidad individual del ítem se mide para indicadores reflectivos examinando las cargas o correlaciones simples de los indicadores con el constructo que pretende medir. En este sentido el valor de la carga debe ser $\geq 0.707$ para que un indicador se acepte como parte 
integrante del constructo. En las escalas empleadas la mayor parte de los indicadores presentan cargas superiores a 0.7, manteniéndose los 36 ítems que contemplan las tres escalas (Figura 2).

La fiabilidad de un constructo permite comprobar la consistencia interna de todos los indicadores al medir el concepto. Para medir este tipo de fiabilidad se decide recurrir a la fiabilidad compuesta por las ventajas que presenta sobre el alfa de cronbach. (Calvo de Mora \& Criado, 2005).

Como puede observarse en la tabla 2 los valores que se obtienen en la fiabilidad compuesta son superiores a 0,7 para todos los constructos, destacando la agresión física directa, la agresión verbal indirecta, el maltrato físico directo y el maltrato verbal indirecto, cuyos valores alcanza el máximo considerado, 1.

Tabla 2. Fiabilidad compuesta de los constructos

\begin{tabular}{lc}
\hline \multicolumn{1}{c}{ CONSTRUCTOS } & $\begin{array}{c}\text { FIABILIDAD } \\
\text { COMPUESTA }\end{array}$ \\
\hline AGRESIÓN FÍSICA & 0,757 \\
\hline Agresión física directa & 1,000 \\
\hline Agresión física indirecta & 0,765 \\
\hline AGRESIÓN MIXTA & 0,754 \\
\hline AGRESIÓN SOCIAL & 0,750 \\
\hline AGRESIÓN VERBAL & 0,791 \\
\hline Agresión verbal directa & 0,822 \\
\hline Agresión verbal indirecta & 1,000 \\
\hline MALTRATO FISICO & 0,784 \\
\hline Maltrato físico directo & 1,000 \\
\hline Maltrato físico indirecto & 0,794 \\
\hline MALTRATO MIXTO & 0,806 \\
\hline MALTRATO SOCIAL & 0,812 \\
\hline MALTRATO VERBAL & 0,849 \\
\hline Maltrato verbal directo & 0,854 \\
\hline Maltrato verbal indirecto & 1,000 \\
\hline OBSERVADORES & 0,911 \\
\hline
\end{tabular}

Fuente: Datos obtenidos con el programa SmartPLS 2.0.

La validez convergente es analizada a través de la varianza extraída media (AVE), se recomiendan valores superiores a 0.50 . Como puede apreciarse en la tabla 3, casi todos los constructos presentan valores superiores al recomendado, a excepción de los constructos agresión física, maltrato físico y observadores que presentan los valores más bajos en este índice aunque cercanos al valor 0,5 . 
Tabla 3. Validez convergente de los constructos

\begin{tabular}{ll}
\hline \multicolumn{1}{c}{ CONSTRUCTOS } & AVE \\
\hline AGRESIÓN FÍSICA & 0,439 \\
\hline Agresión física directa & 1,000 \\
\hline Agresión física indirecta & 0,521 \\
\hline AGRESIÓN MIXTA & 0,612 \\
\hline AGRESIÓN SOCIAL & 0,503 \\
\hline AGRESIÓN VERBAL & 0,558 \\
\hline Agresión verbal directa & 0,698 \\
\hline Agresión verbal indirecta & 1,000 \\
\hline MALTRATO FISICO & 0,476 \\
\hline Maltrato físico directo & 1,000 \\
\hline Maltrato físico indirecto & 0,564 \\
\hline MALTRATO MIXTO & 0,676 \\
\hline MALTRATO SOCIAL & 0,591 \\
\hline MALTRATO VERBAL & 0,653 \\
\hline Maltrato verbal directo & 0,745 \\
\hline Maltrato verbal indirecto & 1,000 \\
\hline OBSERVADORES & 0,460 \\
\hline
\end{tabular}

Fuente: Datos obtenidos con el programa SmartPLS 2.0.

La validez discriminante indica si un constructo es diferente a otro, para ello siguiendo la condición establecida por Fornell \& Larcker (1981), se calcula la raíz cuadrada de la AVE, que ha de ser mayor que las correlaciones que presentan con el resto de constructos. Dichos valores se recogen en la tabla 4, donde los elementos de la diagonal (sombreado) corresponden a la raíz cuadrada de la varianza extraída media del constructo (AVE). El resto de elementos son las correlaciones entre dichos constructos.

Tabla 4. Validez discriminante

\begin{tabular}{|c|c|c|c|c|c|c|c|c|c|}
\hline & $\begin{array}{l}\text { AGRE. } \\
\text { FÍSICA }\end{array}$ & $\begin{array}{l}\text { AGRE. } \\
\text { MIXTA }\end{array}$ & $\begin{array}{l}\text { AGRE. } \\
\text { SOCIAL }\end{array}$ & $\begin{array}{c}\text { AGRE. } \\
\text { VERBAL }\end{array}$ & $\begin{array}{c}\text { MAL. } \\
\text { FISICO }\end{array}$ & $\begin{array}{c}\text { MAL. } \\
\text { MIXTO }\end{array}$ & $\begin{array}{c}\text { MAL. } \\
\text { SOCIAL } \\
\end{array}$ & $\begin{array}{c}\text { MAL } \\
\text { VERBA } \\
\end{array}$ & OBSER \\
\hline AGRE. FÍSICA & 0,662 & & & & & & & & \\
\hline AGRE. MIXTA & 0,540 & 0,783 & & & & & & & \\
\hline AGRE. SOCIAL & 0,400 & 0,413 & 0,710 & & & & & & \\
\hline AGRE. VERBAL & 0,506 & 0,516 & 0,406 & 0,747 & & & & & \\
\hline MAL. FISICO & 0,309 & 0,241 & 0,186 & 0,248 & 0,690 & & & & \\
\hline MAL. MIXTO & 0,251 & 0,316 & 0,210 & 0,254 & 0,518 & 0,822 & & & \\
\hline MAL. SOCIAL & 0,217 & 0,202 & 0,203 & 0,189 & 0,475 & 0,507 & 0,769 & & \\
\hline MAL. VERBAL & 0,216 & 0,236 & 0,188 & 0,350 & 0,547 & 0,564 & 0,549 & 0,808 & \\
\hline OBSER & 0,352 & 0,344 & 0,282 & 0,390 & 0,391 & 0,330 & 0,304 & 0,385 & 0,679 \\
\hline
\end{tabular}

Fuente: Datos obtenidos con el programa SmartPLS 2.0. 
De los resultados obtenidos para este índice, se puede afirmar que existe validez discriminante.

\section{Valoración del modelo estructural}

Se analiza la varianza explicada de las variables endógenas. El valor $\mathrm{R}^{2}$ (coeficiente de determinación) es la cantidad de varianza del constructo que es explicada por el modelo tabla 5 y figura 2 .

Tabla 5. Coeficiente de determinación $\left(R^{2}\right)$ de las variables dependientes

\begin{tabular}{lc}
\hline \multicolumn{1}{c}{ CONSTRUCTOS } & R Square \\
\hline AGRESIÓN FÍSICA & 0,124 \\
\hline Agresión física directa & 0,478 \\
\hline Agresión física indirecta & 0,817 \\
\hline AGRESIÓN MIXTA & 0,118 \\
\hline AGRESIÓN SOCIAL & 0,079 \\
\hline AGRESIÓN VERBAL & 0,152 \\
\hline Agresión verbal directa & 0,820 \\
\hline Agresión verbal indirecta & 0,532 \\
\hline MALTRATO FISICO & 0,188 \\
\hline Maltrato físico directo & 0,452 \\
\hline Maltrato físico indirecto & 0,860 \\
\hline MALTRATO MIXTO & 0,156 \\
\hline MALTRATO SOCIAL & 0,115 \\
\hline MALTRATO VERBAL & 0,196 \\
\hline Maltrato verbal directo & 0,864 \\
\hline Maltrato verbal indirecto & 0,673 \\
\hline
\end{tabular}

Fuente: Datos obtenidos con el programa SmartPLS 2.0.

Falk \& Miller (1992) señalan que la varianza explicada de las variables endógenas $\left(\mathrm{R}^{2}\right)$ debería ser mayor o igual a 0.1 . En nuestro estudio, como se puede observar en la tabla 5 y figura 2, el modelo presenta un adecuado poder predictivo. Todos los constructos dependientes, exceptuando el constructo Agresión social que está próximo a 1, tienen valores de $\mathrm{R}^{2}$ superiores a 0,1 .

Los constructos que presentan un nivel predictivo más bajo, por este orden son: agresión social; maltrato social; agresión mixta; maltrato mixto; agresión física; agresión verbal; maltrato físico y maltrato verbal. Por el contrario se alcanza el máximo poder predictivo para los constructos agresión física directa (47,8\%); maltrato físico directo (45,2 \%); agresión verbal indirecta $(53,2 \%)$; maltrato verbal indirecto $(67,3 \%)$, agresión verbal directa $(82 \%)$ y maltrato verbal directo $(86,4 \%)$.

Los coeficientes path o pesos de regresión estandarizados, se identifican en el nomograma por medio de las flechas que vinculan a los constructos en el modelo interno. 
Chin (1998) propone que para ser considerados significativos, los coeficientes path estandarizados deberían alcanzar al menos un valor de 0.2 , e idealmente situarse por encima de 0.3. Falk \& Miller (1992: 80) proponen una regla empírica, según la cual una variable predictora debería explicar al menos el $1.5 \%$ de la varianza en una variable predicha.

En este sentido como se aprecia en la tabla 6 en el modelo propuesto se observan 12 relaciones en las cuales las variables predictoras explican más del 1,5\% de la varianza de las variables dependientes. Por otro lado se recurre a la técnica de remuestreo Bootstrapping para determinar la significación estadística de los coeficientes paths.

Tabla 6. Descomposición de la varianza

\begin{tabular}{lcccc}
\hline $\begin{array}{c}\text { RELACIONES DEL } \\
\text { MODELO INTERNO }\end{array}$ & $\begin{array}{c}\text { COEF. } \\
\text { PATH }\end{array}$ & CORRELAC. & $\begin{array}{c}\text { \% DE LA } \\
\text { VARIANZA } \\
\text { EXPLICADA }\end{array}$ & P VALUES \\
\hline AGRE FISI $\rightarrow \mathrm{MF}$ & $0,178^{* * * *}$ & 0,309 & $5,5 \%$ & 0.000 \\
\hline AGRE VERBAL $\rightarrow \mathrm{MV}$ & $0,229^{* * *}$ & 0,350 & $8 \%$ & 0.000 \\
\hline AGRE SOCIAL $\rightarrow \mathrm{MS}$ & $0,089^{* * *}$ & 0,203 & $1,8 \%$ & 0,005 \\
\hline AGRE MIXTA $\rightarrow \mathrm{MM}$ & $0,229^{* * * *}$ & 0,316 & $7,2 \%$ & 0.000 \\
\hline OBSERV $\rightarrow \mathrm{MF}$ & $0,313^{* * *}$ & 0,391 & $12,2 \%$ & 0.000 \\
\hline OBSERV $\rightarrow \mathrm{AF}$ & $0,352^{* * *}$ & 0,352 & $12,3 \%$ & 0.000 \\
\hline OBSERV $\rightarrow \mathrm{MV}$ & $0,291^{* * *}$ & 0,385 & $11,2 \%$ & 0.000 \\
\hline OBSERV $\rightarrow \mathrm{AV}$ & $0,390^{* * *}$ & 0,390 & 15,2 & 0.000 \\
\hline OBSERV $\rightarrow \mathrm{AS}$ & $0,282^{* * * *}$ & 0,282 & $7,9 \%$ & 0.000 \\
\hline OBSERV $\rightarrow \mathrm{MS}$ & $0,237^{* * *}$ & 0,304 & $7,2 \%$ & 0.000 \\
\hline OBSERV $\rightarrow \mathrm{AM}$ & $0,344^{* * *}$ & 0,344 & $11,83 \%$ & 0.000 \\
\hline OBSERV $\rightarrow \mathrm{MM}$ & $0,252^{* * *}$ & 0,330 & $8,3 \%$ & 0.000 \\
\hline
\end{tabular}

Para $\mathrm{n}=5.000$ submuestras: ${ }^{*} \mathrm{p}<.05 ;{ }^{* *} \mathrm{p}<.01{ }^{* * *} \mathrm{p}<.001 \mathrm{t}$ de Student de una cola) $\mathrm{t}(0.05 ; 4999)=1,645$; $\mathrm{t}(0.01 ; 4999)=2,327 ; \mathrm{t}(0.001 ; 4999)=3,092$.

Fuente: Datos obtenidos con el programa SmartPLS 2.0.

\section{Discusión y Conclusiones}

El análisis del modelo confirma la influencia que tiene la presencia de los observadores sobre la agresión, en sus distintas manifestaciones $\left(\mathrm{H}_{5}\right.$ : física, verbal, social y mixta) y sobre el sentimiento de maltrato $\left(\mathrm{H}_{6}\right.$ : físico, verbal, social y mixto). Estos resultados vienen a corroborar otras investigaciones (Cuevas \& Marmolejo, 2016; De la Caba-Collado, LópezAtxurra \& Bobowik, 2016; González, Gutiérrez \& Checa, 2017) donde ponen de relieve el papel de los observadores participantes en quienes con sus comportamientos instigan, alientan, apoyan y contribuyen con actos intimidatorios o, en su defecto, realizan acciones para detenerlos. El rol de espectadores/observadores se presenta como un factor revelador para la detección del maltrato escolar. Las respuestas de los espectadores contribuyen a dinámica de acoso al recompensar o sancionar el comportamiento de los perpetradores. Dichas respuestas se pueden cambiar a través de programas escolares que medien sobre los efectos de la intimidación (Saarento \& Salmivalli, 2015).

El papel determinante que juega las conductas de los espectadores en la intimidación y en el ajuste de víctimas, se han venido reconociendo cada vez más en escuelas a través de programas antibullying $(\mathrm{KiVa})$. 
En un estudio reciente sobre mecanismos de mediación del programa antibullying de $\mathrm{KiVa}$, basado en el enfoque del rol del observador participante, influyendo en los compañeros de clase, se ha comprobado cómo se han reducido los comportamientos de intimidación (Saarento, Boulton, \& Salmivalli, 2015).

Por otro lado, el modelo propuesto en esta investigación, parece predecir muy bien, la agresión verbal directa, el maltrato verbal directo, la agresión verbal indirecta, el maltrato verbal indirecto y la agresión física indirecta y el maltrato físico indirecto. En menor medida la agresión física directa y el maltrato físico directo, por lo que pone de relieve que el acoso escolar no sólo consiste en peleas y golpes, quizás se piense que es la forma más común de maltrato escolar, sino que el hostigamiento y el maltrato verbal, en consonancia con ÁlvarezGarcía et al. (2015), se presenta como el tipo de violencia más habitual. Parece que la agresión verbal es algo habitualmente reconocido e incluso tolerable, en los centros educativos pudiendo desencadenar en un futuro en posibles formas de maltrato físico.

Por lo tanto, en educación primaria se presenta la agresión verbal como la forma más habitual de maltrato.

No obstante, todos los tipos de agresiones incluidos en el modelo pronostican, en distinta medida los diferentes tipos de maltrato.

Estos resultados corroboran las evidencias de otros estudios que destacan como tipos de acoso el maltrato físico, verbal, social y mixto (Cuevas \& Marmolejo, 2016; De la CabaCollado et al., 2016; Ruíz, Riuró \& Tesouro, 2015)

Como limitaciones del estudio se puede decir que los resultados quedan restringido a un contexto local y a la propia percepción del alumnado como sujetos implicados en el acoso escolar, por lo que se considera interesante para investigaciones futuras contrastar estos resultados con otros sujetos implicados como pueden ser familiares y profesores y ampliar la muestra a otras localidades. También considerar el modelo como objeto de mejora para lograr un mejor ajuste, examinado con otras técnicas estadísticas (prueba de contraste Chi-cuadrado para la comparación de grupos en función del sexo y curso) si niños y niñas difieren a la hora de definir conductas de acoso.

Por último, señalar que la mayoría de las investigaciones se centran en el estudio del acoso escolar en centros de educación secundaria, esta investigación quiere poner de manifiesto que esta problemática empieza dándose en educación primaria (Albaladejo et al., 2013; Tresgallo, 2011) y se pretende hacer visible la importancia de las primeras edades en el desarrollo del entramado relacional. Asimismo, se destaca la necesidad de actuar a nivel preventivo con el grupo para el adecuado desarrollo social y emocional de cada uno de sus componentes que, en definitiva, influirá en el clima de convivencia del mismo (Sánchez \& Cerezo, 2011).

La información obtenida en este estudio puede servir como punto de partida para diseñar posibles intervenciones educativas diferenciada (víctima y agresores) que favorezcan el tipo de relaciones entre compañeros y las competencias necesarias en los espectadores para que se conviertan en defensores activos (Gini et al. 2008). 


\section{Referencias}

Albaladejo, N., Ferrer, R., Reig, A. \& Fernández M.D. (2013). ¿Existe Violencia Escolar en Educación Infantil y Primaria? Una propuesta para su evaluación y gestión. Anales de psicología, 3(29), 1060-1069.

Álvarez-García, D., Margarita, J., Rodríguez, F.J., \& Núñez, J.C. (2015). Adaptación y validación del cuestionario $\mathrm{CUVE}^{3}$-EP para la evaluación de la violencia escolar en centros de enseñanza básica de la República Dominicana. Anales de Psicología, 31(3), 859-868. Extraído el 16 de Noviembre de 2017 desde https://dx.doi.org/10.6018/analesps.31.3.186482

Calvo de Mora, A. \& Criado, F. (2003). Análisis de la validez del modelo europeo de excelencia para la gestión de la calidad en instituciones universitarias: un enfoque directivo. Revista Europea de Dirección y Economía de la Empresa, 3(14), 41-58.

Cerezo, F., Sánchez, C., Ruiz, C., \& Arense, J.J. (2015). Roles en bullying de adolescentes y preadolescentes, y su relación con el clima social y los estilos educativos parentales. Revista de Psicodidáctica, 20(1), 139-155.

Chin, W.W. (1998). The Partial Least Squares Approach to Structural Equation Modeling, en G.A. Marcoulides [ed.]: Modern Methods for Business Research, 295-336. Mahwah, NJ: Lawrence Erlbaum Associates, Publisher.

Collell, J \& Escudé, C. (2003). Cuestionario para mejorar el conocimiento sobre uno mismo y sobre las relaciones con los demás. Extraído el 16 de Noviembre de 2017 desde http://www.amnistiacatalunya.org/edu/material/webquest/local/bullyingresadir4cuestionario-es.pdf

Cuevas, M.C. \& Marmolejo, M.A. (2016). Observadores: un rol determinante en el acoso escolar. Pensamiento psicológico, 1(14), 89-102.

De la Caba-Collado, M.A., López-Atxurra, R. \& Bobowik, M. (2016). Tareas de responsabilidad social en la escuela y agresión entre iguales. Revista de Educación, 374, 187-210.

Falk, R.F. \& Miller, N.B. (1992). A Primer for Soft Modeling. Akron, Ohio: The University of Akron.

Fornell, C. \& Larcker, D.F. (1981). Evaluating Structural Equation Models with Unobservable Variables and Measurement Error. Journal of Marketing Research, 18, 39-50.

Garaigordobil, M. (2017). Conducta antisocial: conexión con bullying/cyberbullying y estrategias de resolución de conflictos. Psychosocial Intervention, 26, 47-54.

Garaigordobil, M., Martínez-Valderrey, V. \& Machimbarrena, J.M. (2017). Intervención en el bullying y cyberbullying: Evaluación del caso Martín. Revista de Psicología Clínica con Niños y Adolescentes, 1(4), 25-32.

Gini, G., Albiero, P., Benelli, B. \& Altoè, G. (2008). Determinants of adolescents' active defending and passive bystanding behavior in bullying. Journal of Adolescence, 31(1), 93-105.

Gómez Bahillo, C. (Coord.) (2006) Protocolo para la medición de la convivencia en los Centros educativos no universitarios de la Comunidad autónoma de Aragón. Cuestionario para las asociaciones de madres y padres de alumnos. Gobierno de Aragón. Departamento de Educación, Cultura y Deporte. 
González, P.M, Gutiérrez, H. \& Checa, M. (2017). Percepción del maltrato entre iguales en educación infantil y primaria. Revista de Educación, 377, 136-160.

Lera, M.J \& Olías, F. (2002). Cuestionario de nominaciones Sociograma. Extraído el 10 de Noviembre de 2017 desde http://www.psicoeducacion.eu/files/bullying/Instrumentos_bullying.pdf

Magaz, A.M., Chorot, P., Santed, M.A., Valiente, R.M. \& Sandín, B. (2016). Evaluación del bullying como victimización: Estructura, fiabilidad y validez del Cuestionario de Acoso entre Iguales (CAI). Revista de Psicopatología y Psicología Clínica, 2(21), 77-95.

Melero, S. (2017). Intervención cognitivo-conductual en una adolescente víctima de acoso escolar. Revista de Psicología Clínica con Niños y Adolescentes, 2(4), 149-155.

Menesini, E \& Salmivalli, C. (2017) Bullying in schools: the state of knowledge and effective interventions. Psychology, Health \& Medicine, 22, 240-253. Extraído el 13 de Noviembre de 2017 desde http://dx.doi.org/10.1080/13548506.2017.1279740

OMS (2016). INSPIRE: seven strategies for ending violence against children. World Health Organization. Extraído el 10 de Noviembre de 2017 desde http://apps.who.int/iris/bitstream/10665/207717/1/9789241565356-eng.pdf?ua=1

Ortega, R., del Rey, R \& Mora-Merchán, J.A. (2008). Cuestionario sobre Convivencia, Conflictos y Violencia Escolar. Cuestionario del alumnado de Primaria. Extraído el 16 de Noviembre de 2017 desde http://www.uco.es/laecovi/img/recursos/Niqo6GT1bRS2R0u.pdf

Ortega-Ruíz, R., Del Rey, R., \& Casas, J.A. (2016). Evaluar el bullying y el ciberbullying validación española del EBIP-Q y del ECIP-Q. Psicología Educativa, 22(22), 71-9.

Ovejero, A., Smith, P.K. \& Yubero, S. (2013). El acoso escolar y su prevención. Perspectivas internacionales. Madrid: Biblioteca Nueva.

Paulino, L.R, Avilés, J.M., \& Sales Luis, P.J. (2016). Bullying, un problema moral: representaciones de sí mismo y desconexiones morales. Revista de Educación, 373, 9-34.

Peets, K., Pöyhönen, V., Juvonen, J., \& Salmivalli, C. (2015). Classroom Norms of Bullying Alter the Degree to Which Children Defend in Response to Their Affective Empathy and Power. Developmental Psychology, 51 (7). Extraído el 13 de Noviembre de 2017 desde http://dx.doi.org/10.1037/a0039287

Puértolas, A. \& Montiel, I. (2017). Bullying en la educación secundaria: una revisión sobre las características de las víctimas y las víctimas-acosadores y las consecuencias de su victimización. Revista de Victimología, 5, 85-128.

Ruíz, R., Riuró, M. \& Tesouro, M. (2015). Estudio del bullying en el ciclo superior de primaria. Educación XX1, 18(1), 345-368. Extraído el 11 de Noviembre de 2017 desde http://dx.doi.org/10.5944/educxx1.18.1.12384

Saarento, S. \& Salmivalli, C. (2015). The Role of Classroom Peer Ecology and Bystanders' Responses in Bullying. Child Development Perspectives, 9(4), 201-205. Extraído el 13 de Noviembre de 2017 desde https://www.researchgate.net/publication/281237939

Saarento, S., Boulton, A. \& Salmivalli, C. (2015). Reducing bullying and victimization: Student- and classroom-level mechanisms of change. Journal of Abnormal Child Psychology, 43, 61-76. 
Salmivalli, C. (2013). El acoso y el grupo de iguales. En A. Ovejero, P.K. Smith y S. Yubero. El acoso escolar y su prevención. Perspectivas internacionales, pp. 111-130. Madrid: Biblioteca Nueva.

Song, J., \& Oh, I. (2017). Investigation of the bystander effect in school bullying: Comparison of experiential, psychological and situational factors. School Psychology International, 38(3), 319-336.

Tresgallo, E. (2011). El acoso escolar y las actitudes psicológicas defensivas en el primer ciclo de Educación Primaria. Revista Española de Orientación y Psicopedagogía, 22(1), 2837.

Zych, I, Ortega-Ruiz, R., \& Marín-López, I. (2016). Cyberbullying: a systematic review of research, its prevalence and assessment issues in Spanish studies. Psicología Educativa, $22,5-18$. 